\title{
A bypass of an arrow is sectional
}

\author{
By
}

\author{
William Crawley-Boevey, Dieter Happel and Claus Michael Ringel
}

Given a vertex $y$ in a quiver, we denote by $y^{+}$the set of vertices $z$ with an arrow $y \rightarrow z$, and by $y^{-}$the set of vertices $x$ with an arrow $x \rightarrow y$. Let $\Gamma=\left(\Gamma_{0}, \Gamma_{1}, \tau\right)$ be a translation quiver, thus $\left(\Gamma_{0}, \Gamma_{1}\right)$ is a (locally finite) quiver without multiple arrows, and $\tau: \Gamma_{0}^{\prime} \rightarrow \Gamma_{0}$ is an injective map, where $\Gamma_{0}^{\prime}$ is a subset of $\Gamma_{0}$, such that for any $z \in \Gamma_{0}^{\prime}$ we have $z^{-}=(\tau z)^{+}$. A vertex of $\Gamma$ which does not belong to $\Gamma_{0}^{\prime}$ is said to be projective, one which does not belong to $\tau\left(\Gamma_{0}^{\prime}\right)$ is said to be injective. Recall that a path $y_{0} \rightarrow y_{1} \rightarrow \cdots \rightarrow y_{n}$ in $\Gamma$ is said to be sectional provided for every $0<i<n$, we have $\tau y_{i+1} \neq y_{i-1}$. It is called cyclic if $y_{0}=y_{n}$ and $n \geqq 1$. We consider the following conditions:

(NC) There is no cyclic path.

(PQ) If $x_{0} \rightarrow p$ is an arrow, with $p$ projective, and $x_{0} \rightarrow x_{1} \rightarrow \cdots \rightarrow x_{n}=q$ is a sectional path, with $q$ injective, then $n \geqq 1$, and $p=x_{1}$.

If $x \rightarrow z$ is an arrow in a quiver without cyclic paths, any path $x=y_{0} \rightarrow y_{1} \rightarrow \cdots \rightarrow y_{n}$ $=z$ of length $n \geqq 2$ will be called a bypass for $x \rightarrow z$.

If $x \rightarrow z$ is an arrow in a translation quiver any sectional path $x=y_{0} \rightarrow y_{1} \rightarrow \cdots \rightarrow y_{n}$ $=z$ of length $n \geqq 2$ will be called a sectional bypass for $x \rightarrow z$, provided we have in addition $y_{1} \neq y_{n}, y_{0} \neq y_{n-1}$.

Proposition 1. Assume the conditions (NC) and (PQ) are satisfied. Then any bypass of an arrow is sectional.

P r o of. Let $x \rightarrow z$ be an arrow, and $x=y_{0} \rightarrow y_{1} \rightarrow \cdots \rightarrow y_{n}=z$ a bypass, and assume it is not sectional.

Consider first the case when $z$ is projective. We have $y_{1} \neq z$, since otherwise we would have a cyclic path. Take $r$ maximal with $0<r<n$, such that the path $y_{0} \rightarrow y_{1} \rightarrow \cdots \rightarrow y_{r}$ is sectional. The condition (PQ) asserts that none of the vertices $y_{i}$, with $0 \leqq i \leqq r$ can be injective, since $y_{1} \neq z$. Therefore, we can form the vertices $\tau^{-} y_{i}$, and we do this for $0 \leqq i \leqq r-1$. We obtain a path $z \rightarrow \tau^{-} y_{0} \rightarrow \tau^{-} y_{1} \rightarrow \cdots \rightarrow \tau^{-} y_{r-1}=y_{r+1}$ of length $r \geqq 1$, which we can compose with the given path from $y_{r+1}$ to $y_{n}=z$ in order to obtain a cyclic path, in contradiction to (NC).

Assume now that $z$ is not projective. We have $x \neq y_{n-1}$, since otherwise we would have a cyclic path. Take $s$ minimal with $0<s<n$, such that the path $y_{s} \rightarrow y_{s+1} \rightarrow \cdots \rightarrow y_{n}$ is sectional, therefore $\tau y_{s+1}=y_{s-1}$. 
Consider the case where one of the vertices $y_{t}$ with $s+1<t<n$ is projective, and take $t$ maximal with this property. We can form $\tau y_{i}$ for $t+1 \leqq i \leqq n$, and we obtain a path $\tau y_{t+1} \rightarrow \cdots \rightarrow \tau y_{n} \rightarrow x$. If we compose this path with the given path $x=y_{0} \rightarrow y_{1} \rightarrow \cdots \rightarrow y_{t}$, then we have a bypass for the arrow $\tau y_{t+1} \rightarrow y_{t}$. On the one hand, this bypass is not sectional, since it passes through $y_{s-1} \rightarrow y_{s} \rightarrow y_{s+1}$, on the other hand, it ends in the projective vertex $y_{t}$. But we have seen already that this is impossible.

It follows that none of the vertices $y_{i}$, with $\mathrm{s}+1 \leqq i<n$ is projective, thus we can form $\tau y_{i}$, for these $i$, and we obtain a path $\tau y_{t+1} \rightarrow \cdots \rightarrow \tau y_{n} \rightarrow x$ of length $n-t \geqq 1$. We compose this with the given path from $x=y_{0}$ to $y_{t-1}=\tau y_{t+1}$ and obtain in this way a cyclic path, in contradiction to (NC). This completes the proof.

Recall that a function $f: \Gamma_{0} \rightarrow \mathbb{N}_{1}$ is said to be subadditive, provided $f(\tau z)+f(z)$ $\geqq \sum_{y \in z^{-}} f(y)$, for every non-projective $z$. The following conditions will be of interest:

$\left(\mathbf{P}_{\leqq}\right)$If $y \rightarrow p$ is an arrow, and $p$ is projective, then $f(y) \leqq f(p)$.

$\left(\mathrm{P}_{<}\right)$If $y \rightarrow p$ is an arrow, and $p$ is projective, then $f(y)<f(p)$.

$\left(\mathrm{Q}_{\geqq}\right)$If $q \rightarrow y$ is an arrow, and $q$ is injective, then $f(q) \geqq f(y)$.

$\left(\mathrm{Q}_{>}\right)$If $q \rightarrow y$ is an arrow, and $q$ is injective, then $f(q)>f(y)$.

(A) If $x \rightarrow y$ is an arrow, then $f(x) \neq f(y)$.

Of course, under the condition $(\mathrm{A})$, the conditions $\left(\mathrm{P}_{<}\right)$and $\left(\mathrm{P}_{\leqq}\right)$coincide, and similarly also $\left(Q_{>}\right)$and $\left(Q_{\geqq}\right)$.

Lemma. Assume there exists a subadditive function $f: \Gamma_{0} \rightarrow \mathbb{N}_{1}$ which satisfies the conditions $\left(\mathrm{P}_{\leqq}\right)$and $\left(\mathrm{Q}_{>}\right)$. Then the condition $(\mathrm{PQ})$ holds.

Proof. Let $x_{0} \rightarrow p$ be an arrow, with $p$ projective, and $x_{0} \rightarrow x_{1} \rightarrow \cdots \rightarrow x_{n}=q$ a sectional path, with $q$ injective. If $n=0$, then we deal with an arrow $q \rightarrow p$. However the condition ( $\left.\mathbf{P}_{\leqq}\right)$asserts $f(q) \leqq f(p)$, whereas the condition $\left(\mathrm{Q}_{>}\right)$yields $f(q)>f(p)$. Thus, we must have $n \geqq 1$. Assume we have $p \neq x_{1}$. We can assume that none of the vertices $x_{i}$ with $0 \leqq i<n$ is injective. Denote $y_{0}=p$, and, $y_{i}=\tau^{-} x_{i-1}$, for $1 \leqq i \leqq n$. Then, for $0 \leqq i<n$, the set $x_{i}^{+}$contains the vertices $y_{i}$ and $x_{i+1}$, and they are always different, thus the subadditivity gives $f\left(x_{i}\right)+f\left(y_{i+1}\right) \geqq f\left(y_{i}\right)+f\left(x_{i+1}\right)$ for these $i$. We rewrite this as $f\left(x_{i}\right)-f\left(x_{i+1}\right) \geqq f\left(y_{i}\right)-f\left(y_{i+1}\right)$, add up, and obtain $f\left(x_{0}\right)+f\left(y_{n}\right) \geqq f\left(y_{0}\right)+f\left(x_{n}\right)$. But $y_{0}$ is projective, thus $f\left(x_{0}\right) \leqq f\left(y_{0}\right)$, and $x_{n}$ is injective, thus $f\left(x_{n}\right)>f\left(y_{n}\right)$. So we obtain a contradiction.

Note that the condition (PQ) is selfdual: if it is satisfied in $\Gamma$, then also in the opposite of $\Gamma$. Thus (PQ) also follows from the conditions $\left(\mathrm{P}_{<}\right)$and $\left(\mathrm{Q}_{\geqq}\right)$.

Ex a m ples. First of all, the conditions $(\mathrm{NC}),\left(\mathrm{P}_{\leqq}\right),\left(\mathrm{Q}_{\geqq}\right)$are not sufficient to enforce that bypasses of arrows are sectional. Take the translation quiver with vertices $x, y, a, b, c$ and arrows $x \rightarrow y, x \rightarrow a, a \rightarrow b, b \rightarrow c, c \rightarrow y$, with $\tau c=a$, and $f(b)=2$, whereas $f(z)=1$ for the remaining vertices. Then $x \rightarrow y$ has a bypass which is not sectional.

Second, the translation quiver $\mathbb{Z} \Delta$, where $\Delta$ has three vertices $a, b, c$ and arrows $a \rightarrow b, a \rightarrow c, b \rightarrow c$. Then there is a sectional path $(0, a) \rightarrow(0, c) \rightarrow(1, b)$, and the 
non-sectional path $(0, a) \rightarrow(0, b) \rightarrow(1, a) \rightarrow(1, b)$. We see that even in a stable translation quiver without cyclic paths, a bypass of a sectional path of length two does not have to be sectional.

We consider now translation quivers which may have cyclic paths. The following is a special case of considerations in [1].

Proposition 2. Let $\Gamma$ be a translation quiver, and assume there exists a bounded subadditive function $f$ which satisfies the conditions $(\mathrm{A}),\left(\mathrm{P}_{<}\right)$and $\left(\mathrm{Q}_{>}\right)$. Then no arrow has a sectional bypass.

Proof. Assume $y_{0} \rightarrow y_{1} \rightarrow \cdots \rightarrow y_{n}$ is a sectional bypass to the arrow $y_{0} \rightarrow y_{n}$. We consider the case $f\left(y_{0}\right)<f\left(y_{n}\right)$, the remaining case $f\left(y_{0}\right)>f\left(y_{n}\right)$ follows by duality.

Because of $f\left(y_{0}\right)<f\left(y_{n}\right)$, the vertex $y_{0}$ cannot be injective, thus we can form $y_{n+1}=\tau^{-} y_{0}$. There are arrows $y_{1} \rightarrow y_{n+1}$ and $y_{n} \rightarrow y_{n+1}$. By definition we have $y_{0} \neq y_{n-1}$, thus the path $y_{1} \rightarrow y_{2} \rightarrow \cdots \rightarrow y_{n} \rightarrow y_{n+1}$ is sectional. Now $y_{1} \neq y_{n}$ by the definition of a sectional bypass, and $y_{2} \neq y_{n+1}$, since the original path was sectional. Therefore $y_{1} \rightarrow y_{2} \rightarrow \cdots \rightarrow y_{n} \rightarrow y_{n+1}$ is a sectional bypass to the arrow $y_{1} \rightarrow y_{n+1}$. Also, $y_{1} \neq y_{n}$, therefore $f\left(y_{0}\right)+f\left(y_{n+1}\right) \geqq f\left(y_{1}\right)+f\left(y_{n}\right)$, thus $f\left(y_{n+1}\right)-f\left(y_{1}\right) \geqq f\left(y_{n}\right)$ $-f\left(y_{0}\right)>0$. Inductively, we obtain in this way an infinite sequence of vertices $y_{i}$, with $i \in \mathbb{N}_{0}$, such that for all $i$ we have $f\left(y_{n+i}\right)-f\left(y_{i}\right) \geqq f\left(y_{n}\right)-f\left(y_{0}\right)$. It follows that $f$ cannot be bounded.

A p plic a tion. The Auslander-Reiten quiver $\Gamma(A)$ of an Artin algebra $A$ (see e.g. [3]) has as vertices the isomorphism classes of the indecomposable modules, there is an arrow $[X] \rightarrow[Y]$ provided there exists an irreducible map, and $\tau$ is the Auslander-Reiten translation. Of course, the length function is subadditive, and satisfies conditions $\left(P_{<}\right),\left(Q_{>}\right)$ and (A). Thus, if $\mathscr{C}$ is a component of an Auslander-Reiten quiver which has no cycles, then any bypass of an arrow in $\mathscr{C}$ is sectional. This can be used for many components, since according to Zhang [4], a component without projective or injective vertices which is not a tube has no cyclic path.

If $A$ is representation-finite (i.e. $\Gamma(A)$ is finite), Proposition 2 implies that an irreducible map does not allow a sectional bypass.

Corollary. Let $A$ be a representation-directed algebra (i.e. $\Gamma(\Lambda)$ is finite and satisfies (NC)). If $\alpha: X \rightarrow Y$ is an irreducible map between indecomposable A-modules, then $\alpha$ has no bypass.

E $x$ a m p le. Let us comment on the definition of a sectional bypass. Consider the following algebra given as quiver with relation by:

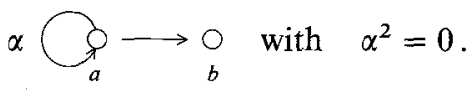


We denote the indecomposable modules by their Loewy-series. Then the AuslanderReiten quiver is given as follows, where the horizontal dotted lines indicate the Auslander-Reiten translation, while identification is along the vertical dashed lines.

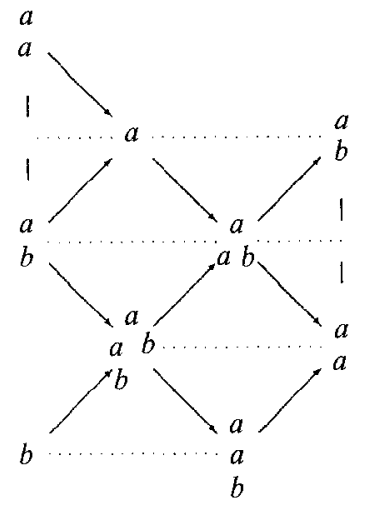

We obtain a sectional path

$$
b \rightarrow \underset{b}{a} b \rightarrow \underset{a}{a} b \rightarrow \underset{b}{a} \rightarrow \underset{b}{a} b
$$

(the first map is the inclusion map of a radical summand, and the second map is surjective). Since we require $y_{1} \neq y_{n}$, this is not a sectional bypass to the first arrow.

We say that a cyclic path $y_{0} \rightarrow y_{1} \rightarrow \cdots \rightarrow y_{n}=y_{0}$ is a sectional cycle if it is sectional and $\tau y_{1} \neq y_{n-1}$. The last example shows that one has to be careful when speaking about sectional cycles. The last three arrows form a sectional path which is cyclic, but it is not a sectional cycle. So the result in [2] should be formulated that the Auslander-Reiten quiver of a representation-finite algebra does not contain a sectional cycle.

\section{References}

[1] R. BAUTISTA and S. BRENNER, Replication numbers for non-Dynkin sectional subgraphs in finite Auslander-Reiten quivers and some properties of Weyl roots. Proc. London Math. Soc. (3) 47, $429-462$ (1983).

[2] R. Bautista and S. Smalø, Nonexistent cycles. Comm. Algebra (16) 11, 1755-1767 (1983).

[3] C. M. RingeL, Tame algebras and integral quadratic forms. LNM 1099, Berlin-Heidelberg-New York 1984.

[4] Y. ZHANG, The structure of stable components. Canad. J. Math., to appear.

Eingegangen am 13.2. 1991

Anschriften der Autoren:
W. Crawley-Bocvey
D. Happel, C. M. Ringel
Mathematical Institute
Oxford University
Fakultät für Mathematik
24-29 St. Giles
Universität Bielefeld
Oxford OX1 3LB, England
Postfach 8640
DW-4800 Bielefeld 1 